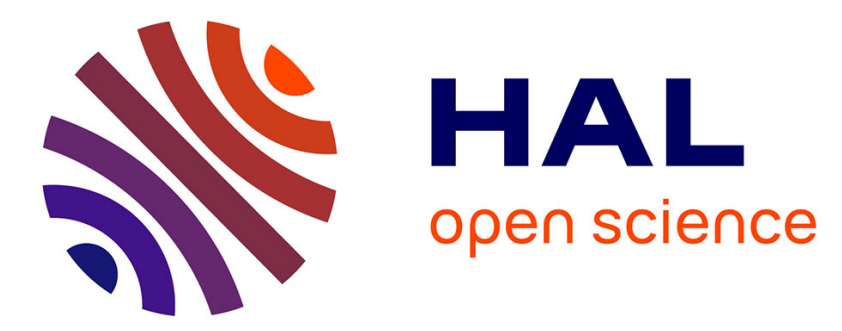

\title{
Automated Categorization of Parkinsonian Syndromes Using Magnetic Resonance Imaging in a Clinical Setting
}

Lydia Chougar, Johann Faouzi, Nadya Pyatigorskaya, Lydia Yahia-cherif, Rahul Gaurav, Emma Biondetti, Marie Villotte, Romain Valabregue, Jean-christophe Corvol, Alexis Brice, et al.

\section{To cite this version:}

Lydia Chougar, Johann Faouzi, Nadya Pyatigorskaya, Lydia Yahia-cherif, Rahul Gaurav, et al.. Automated Categorization of Parkinsonian Syndromes Using Magnetic Resonance Imaging in a Clinical Setting. Movement Disorders, 2020, 10.1002/mds.28348 . hal-03046578v2

\section{HAL Id: hal-03046578 \\ https://hal.sorbonne-universite.fr/hal-03046578v2}

Submitted on 8 Dec 2020

HAL is a multi-disciplinary open access archive for the deposit and dissemination of scientific research documents, whether they are published or not. The documents may come from teaching and research institutions in France or abroad, or from public or private research centers.
L'archive ouverte pluridisciplinaire HAL, est destinée au dépôt et à la diffusion de documents scientifiques de niveau recherche, publiés ou non, émanant des établissements d'enseignement et de recherche français ou étrangers, des laboratoires publics ou privés. 


\section{Automated Categorization of Parkinsonian Syndromes Using Magnetic Resonance Imaging in a Clinical Setting}

Lydia Chougar ${ }^{1,2,3,4}$, MD; Johann Faouzi ${ }^{1,5}$; Nadya Pyatigorskaya ${ }^{1,2,3,4}$, MD, PhD; Lydia Yahia-Cherif ${ }^{1,3}$, PhD; Rahul Gaurav ${ }^{1,2,3}$; Emma Biondetti ${ }^{1,2,3}$, PhD; Marie Villotte $^{6}, \mathrm{MD}$; Romain Valabrègue ${ }^{1,3}, \mathrm{PhD}$; Jean-Christophe Corvol ${ }^{1,7}, \mathrm{MD}, \mathrm{PhD}$; Alexis Brice ${ }^{1,8}$, MD, PhD; Louise-Laure Mariani ${ }^{1,7,9}$, MD, PhD; Florence Cormier ${ }^{9}$, MD; Marie Vidailhet ${ }^{1,2,9}$, MD, PhD; Gwendoline Dupont ${ }^{10,11}$, MD; Ines Piot ${ }^{4}$, MD; David Grabli $^{9}$, MD, PhD; Christine Payan ${ }^{12,13}$, MD; Olivier Colliot ${ }^{1,5}$, PhD; Bertrand Degos $^{14,15}$, MD, PhD; Stéphane Lehéricy ${ }^{1,2,3,4}, \mathrm{MD}, \mathrm{PhD}$

${ }^{1}$ Paris Brain Institute - ICM, Inserm U 1127, CNRS UMR 7225, Sorbonne Université, UMR $S$ 1127, CNRS UMR 7225, Paris, France

${ }^{2}$ ICM, "Movement Investigations and Therapeutics" Team (MOV'IT), Paris, France

${ }^{3}$ ICM, Centre de NeuroImagerie de Recherche - CENIR, Paris, France

${ }^{4}$ Department of Neuroradiology, Pitié-Salpêtrière University Hospital, APHP, Paris, France

${ }^{5}$ Inria, Aramis Team, Paris, France

${ }^{6}$ Faculté de Médecine, Université Denis Diderot, Paris, France

7 ICM, Centre d'Investigation Clinique Neurosciences, Paris, France

${ }^{8}$ ICM, Team Neurogénétique Fondamentale et Translationnelle, Paris, France

${ }^{9}$ Clinique des Mouvements Anormaux, Département des Maladies du Système Nerveux, Hôpital Pitié-Salpêtrière, APHP, Paris, France

${ }^{10}$ Université de Bourgogne, Dijon, France

${ }^{11}$ Centre Hospitalier Universitaire François Mitterrand, Département de Neurologie, Dijon,

France

12 BESPIM, Hôpital Universitaire de Nîmes, Nîmes, France

13 Service de Pharmacologie Clinique, Hôpital Pitié-Salpêtrière, APHP, Paris, France

${ }^{14}$ Dynamics and Pathophysiology of Neuronal Networks Team, Center for Interdisciplinary

Research in Biology, Collège de France, CNRS UMR 7241 / Inserm U1050, MemoLife Labex, Paris, France

${ }^{15}$ Department of Neurology, Avicenne University Hospital, Sorbonne Paris Nord University, Bobigny, France

Journal: Movement Disorders

First published: 02 November 2020

DOI: https://doi.org/10.1002/mds. 28348 


\section{Abstract}

Background: Machine learning algorithms using magnetic resonance imaging (MRI) data can accurately discriminate parkinsonian syndromes. Validation in patients recruited in routine clinical practice is missing.

Objective: The aim of this study was to assess the accuracy of a machine learning algorithm trained on a research cohort and tested on an independent clinical replication cohort for the categorization of parkinsonian syndromes.

Methods: Three hundred twenty-two subjects, including 94 healthy control subjects, 119 patients with Parkinson's disease (PD), 51 patients with progressive supranuclear palsy (PSP) with Richardson's syndrome, 35 with multiple system atrophy (MSA) of the parkinsonian variant (MSA-P), and 23 with MSA of the cerebellar variant (MSA$\mathrm{C})$, were recruited. They were divided into a training cohort $(n=179)$ scanned in a research environment and a replication cohort $(n=143)$ examined in clinical practice on different MRI systems. Volumes and diffusion tensor imaging (DTI) metrics in 13 brain regions were used as input for a supervised machine learning algorithm. To harmonize data across scanners and reduce scanner-dependent effects, we tested two types of normalizations using patient data or healthy control data.

Results: In the replication cohort, high accuracies were achieved using volumetry in the classification of PD vs PSP, PD vs MSA-C, PSP vs MSA-C, and PD vs atypical parkinsonism (balanced accuracies: $0.840-0.983$, area under the receiver operating characteristic curves: $0.907-0.995)$. Performances were lower for the classification of PD vs MSA-P, MSA-C vs MSA-P (balanced accuracies: $0.765-0.784$, area under the receiver operating characteristic curve: $0.839-0.871$ ) and PD vs PSP vs MSA (balanced accuracies: 0.773). Performance using DTI was improved when normalizing by controls, but remained lower than that using volumetry alone or combined with DTI.

Conclusions: A machine learning approach based on volumetry enabled accurate classification of subjects with early-stage parkinsonism, examined on different MRI systems, as part of their clinical assessment.

Key Words: Parkinson's disease; progressive supranuclear palsy; multiple system atrophy; multimodal magnetic resonance imaging; machine learning algorithm 


\section{Introduction}

Diagnosis of idiopathic Parkinson's disease (PD) and atypical parkinsonism, whose most frequent types are progressive supranuclear palsy (PSP) and multiple system atrophy (MSA), relies on clinical criteria (Gilman et al., 2008; Hoglinger et al., 2017; Hughes et al., 2002). The diagnostic accuracy of PD is greatly improved when made by clinical experts in movement disorders with a sensitivity of $91.1 \%$ and a specificity of $98.4 \%$ (Berardelli et al., 2013; Hughes et al., 2002). However, on initial presentation, a correct diagnosis of atypical parkinsonism may be difficult, and clinical uncertainty is high. Accurate diagnosis is crucial to assess the prognosis, enroll patients in adequate care systems, and allow their inclusion in appropriate therapeutic trials.

Degeneration of dopaminergic neurons within the substantia nigra pars compacta is the hallmark of neurodegenerative parkinsonian syndromes (Dickson, 2012, 2018; Levin et al., 2016). Although patients with PD exhibit only nigral abnormalities in a limited number of small brain stem nuclei at the early stage of the disease (Dickson, 2012, 2018; Kalia and Lang, 2015; Levin et al., 2016), patients with PSP show a larger involvement of the midbrain, dentate nucleus, and superior cerebellar peduncles (SCPs) (Dickson, 2012; Hauw et al., 1994; Levin et al., 2016; Pyatigorskaya et al., 2020; Steele et al., 2014), and patients with MSA are characterized by damage particularly affecting the posterior putamen in the parkinsonian variant (MSA-P) and the pons, middle cerebellar peduncles, and cerebellum in the cerebellar variant (MSA-C) (Castellani, 1998; Dickson, 2012; Levin et al., 2016).

Multimodal magnetic resonance imaging (MRI) can detect these different patterns of brain damage (Heim et al., 2018; Kassubek, 2018; Lehericy et al., 2017; Rizzo et al., 2016; Whitwell et al., 2017). Atrophy is visible on $\mathrm{T}_{1}$-weighted images, tissue microstructure alterations are detected using diffusion-weighted images, and iron deposition can be evidenced using iron-sensitive sequences (Heim et al., 2018; Kassubek, 2018; Lehericy et al., 2017; Rizzo et al., 2016; Whitwell et al., 2017). Using MRI data, machine learning algorithms can accurately differentiate between parkinsonian syndromes (Archer et al., 2019; Cherubini et al., 2014; Du et al., 2017; Huppertz et al., 2016; Morisi et al., 2018; Péran et al., 2018; Salvatore et al., 2014; Scherfler et al., 2016). Most studies have used a single type of MRI data, either volumetry (Huppertz et al., 2016; Salvatore et al., 2014; Scherfler et al., 2016) or diffusion-weighted data (Archer et al., 2019). Some studies have combined volumetry and diffusion (Cherubini et al., 2014), or have included R2* relaxometry (Péran et al., 2018) or spectroscopy (Morisi et al., 2018). Two studies have included large cohorts of 1002 subjects (Archer et al., 2019) or 464 subjects (Huppertz et al., 2016), whereas most studies have investigated smaller samples (Morisi et al., 2018; Salvatore et al., 2014; Scherfler et al., 2016). Only two studies have included subjects with PD, PSP, MSA-P, and MSA-C (Huppertz et al., 2016; Morisi et al., 2018), while other studies have not differentiated between MSA-P and MSA-C (Scherfler et al., 2016), 
have included only patients with MSA-P (Archer et al., 2019) or MSA-C (Du et al., 2017), or have not included patients with MSA (Cherubini et al., 2014; Salvatore et al., 2014) or PSP (Péran et al., 2018). Moreover, these studies have been mainly designed in a research environment and have been tested without an independent replication cohort (Cherubini et al., 2014; Huppertz et al., 2016; Péran et al., 2018; Salvatore et al., 2014). To transpose an automated MRI classification approach based on machine learning to clinical practice, it is necessary to evaluate this approach on large cohorts scanned in clinical environments and including the different types of parkinsonian syndromes.

Our objective was to assess the predictive performance of machine learning algorithms for the categorization of parkinsonian syndromes, including PSP, MSA-C, and MSA-P, compared with PD and healthy control subjects (HCs). Such algorithms were trained on a research cohort and tested on an independent replication cohort scanned on different MRI scanners in clinical conditions in a neuroradiology department, using volumetry and diffusion tensor imaging (DTI).

\section{Materials and Methods}

\subsection{Population}

Two populations of participants were included: a training cohort to train and validate the algorithms and a replication cohort to independently evaluate each algorithm's performance. The training cohort was constituted of research studies conducted between 2007 and 2012 at the Paris Brain Institute (ICM): Genepark (LSHB-CT-2006-037544), BBBIPPS (DGS 2006/0524), and Nucleipark (RCB 2009-A00922-55). Inclusion criteria for patients were a diagnosis of PD, PSP, or MSA established by movement disorders specialists according to published consensus criteria for PD (Hughes et al., 2002) with no or minimal cognitive disturbances with Mini Mental State Examination score $>24$, PSP with Richardson's syndrome (Litvan et al., 1996), or MSA (Gilman et al., 2008).

Participants in the replication cohort were consecutively enrolled between 2013 and 2019 in the movement disorders clinic of the Pitié-Salpêtrière Hospital, Paris. Diagnosis of probable PD, PSP, or MSA was retrospectively established in 2019 by movement disorders specialists according to the aforementioned clinical criteria based on all available clinical data.

The clinical examination included the Unified Parkinson's Disease Rating Scale Part III (UPDRS III) scores. Baseline MRI scans were obtained on the same days as the clinical examination. For both cohorts, HCs without a history of neurological or psychiatric disease were included. Subjects were excluded if they had any additional neurological disorder.

Local institutional review boards approved the studies (Genepark: CPP Paris II, 2007-A00208-45; BBBIPPS: CPP Paris VI, P040410-65-06; Nucleipark: CPP Paris VI, 
65-09; Park Atypique: CPP Ile-de-France VI, 08012015). Written informed consent was obtained from all participants.

\subsection{Image Acquisition}

Participants in the training cohort were scanned at the Paris Brain Institute using a 3T Siemens Trio system (Siemens Healthineers, Erlangen, DE) with a 32-channel head coil. Participants in the replication cohort were scanned in clinical conditions for diagnostic purposes in the Neuroradiology Department of the hospital using two MRI systems: (i) a 3T GE Signa HDxt (GE Healthcare, Chicago, IL) with an 8-channel head coil, and (ii) a 3T Siemens Skyra using a 64-channel head coil. HCs in the replication cohort were scanned twice during different sessions on Siemens Skyra and GE Signa systems. All participants were scanned using a standardized protocol including a highresolution $\mathrm{T}_{1}$-weighted gradient-recalled echo sequence (magnetization-prepared rapid acquisition with gradient-recalled echo or spoiled gradient recalled acquisition in steady state) and DTI with 30 (Siemens Skyra and GE Signa), 60, or 64 (Siemens Trio) diffusion directions. Acquisition parameters are provided in Supporting Information Table S1. Quality control was performed by visual inspection; $\mathrm{T}_{1}$-weighted and diffusion-weighted images with significant motion artifacts or image distortions were excluded.

\subsection{Data Processing and Analysis}

Image processing and analysis were performed using Matlab (R2017b; The MathWorks, Inc., Natick, MA). $\mathrm{T}_{1}$-weighted images were automatically segmented using FreeSurfer 6.0 (http://freesurfer.net/; MGH, Boston, MA, USA) (Fischl et al., 2002). DTI preprocessing was performed using the FMRIB Software Library v5.0 (FMRIB, Oxford, United Kingdom). Motion and eddy currents were corrected using the eddycor function. Fractional anisotropy (FA) and diffusivity maps were computed using the DTIfit function for the entire brain volume. The diffusion maps were coregistered to the threedimensional $\mathrm{T}_{1}$-weighted volume using the Statistical Parametric Mapping coregister function (SPM 12; https://www.fil.ion.ucl.ac.uk/spm/software/spm12/).

We included 13 regions of interest known for being involved in parkinsonian syndromes in the gray matter (midbrain, pons, putamen, posterior putamen, caudate, thalamus, pallidum, precentral cortex, insular cortex), white matter (SCPs, cerebellum white matter including the middle cerebellar peduncles), and ventricles (third ventricle, fourth ventricle). Volumes, average values of FA, mean diffusivity, axial diffusivity, and radial diffusivity $(\mathrm{RD})$ were calculated in all segmented regions of interest and used as input features for the algorithms. To remove inter-individual variability, all volumes

were corrected by the total intracranial volume for each participant. Means of the volumes and DTI metrics were used for bilateral regions. 


\subsection{Normalization Procedures}

Two independent normalization procedures were investigated and performed in both cohorts. "Normalization 1" scaled the features so that they were normally distributed. For a given between-group comparison (e.g. PSP vs PD), each variable of each participant was normalized using the mean and standard deviation (SD) of this variable in all patients in these two groups of the training cohort (e.g. all patients with PSP and $\mathrm{PD}$ in the training cohort). For example, for the "PD vs PSP" classification in either the training or the replication cohort, each variable of each patient with PD and PSP in the corresponding cohort was normalized using the following formula: (Variable vs mean of PD and PSP in the training cohort)/SD of PD and PSP in the training cohort.

"Normalization 2" aimed to harmonize data across scanners, reduce scannerdependent effects in the images, and find a good trade-off between standard normal distribution and same distribution of the features in both cohorts. Normalization 2 used the mean and SD of the controls scanned using the same scanner as the patient, according to the formula: (Variable vs mean of controls scanned using the same scanner)/SD of controls scanned using the same scanner.

\subsection{Machine Learning Algorithms}

Using the scikit-learn package (Pedregosa et al., 2011), four supervised machine learning algorithms were used: logistic regression, support vector machine (SVM) with a linear kernel, SVM with a radial basis function kernel, and random forest. They were trained and validated on the training cohort, then tested on the replication cohort.

The cross-validation procedure on the training cohort included two nested loops: an outer loop with repeated stratified random splits with 50 repetitions evaluating the classification performances and an inner loop with 5-fold cross-validation used to optimize the hyper-parameters of the algorithms. One model was created for each split, leading to 50 models. We selected the model with the highest mean balanced accuracies among the models with frequencies of appearance greater than 10\% (Supporting Information Fig. S1).

Correlation between the different input features was investigated in both cohorts (Supporting Information Tables S2 and S3). Ridge regularization was used to avoid overfitting and to deal with possible correlation between the features. Three models were evaluated: "volumetry only", "DTI only" and "volumetry + DTI" with age and sex as covariates. DTI was missing in 13 patients of the training cohort and 14 patients of the replication cohort. Missing values were imputed with the mean values of the features from the training cohort. The algorithms were retrained and retested after removing subjects with missing DTI images. In addition, analysis with UPDRS III scores alone was performed, and performances were compared with the other models.

We evaluated binary classification tasks (PD vs PSP, PD vs MSA-P, PD vs MSA-C, 
PSP vs MSA-P, PSP vs MSA-C, MSA-P vs MSA-C, PD vs atypical parkinsonism) and the multi-class classification task (PD vs PSP vs MSA, with patients with MSA merged in one group because of their small number).

Receiver operating characteristic curves were generated, and balanced accuracy (BA), area under the curve (AUC), sensitivity, and specificity were calculated to evaluate the algorithm performances. BA was defined as the average sensitivity and specificity in each group. BA avoids overestimation of classification performance because of imbalanced group sizes (Huppertz et al., 2016).

\subsection{Weighting Factors}

Weighting factors of the volume and DTI metrics of each brain region were extracted from the logistic regression training after normalization 2 (corresponding to the best model). They reflected the contribution of each feature to group differentiation. A rescaling to a range of -1 to +1 was applied to highlight the relative importance of each feature: the higher the absolute value, the bigger the contribution of the feature. This assertion relied on the assumption that each feature had the same scale, which was a reasonable assumption because all features were standardized. When the coefficient was positive, the algorithm favored the first group if the value of the feature was high or the second group if the value was low, and vice versa (Huppertz et al., 2016).

\section{7 $\quad$ Statistical Analyses}

\subsubsection{Participant Characteristics}

Statistical analyses were performed using R software (R Core Development Team, 2017). Group demographic and clinical scores within each cohort were analyzed. Statistical difference in sex distribution was evaluated using Fisher's exact test, followed by pairwise comparisons with $p$ adjustment (Holm's method). Age, disease duration, and UPDRS III scores were compared using the Kruskal-Wallis test, followed by pairwise comparisons with Holm-Bonferroni correction. Inter-cohort comparisons were also performed by assessing group-wise differences using Fisher's exact test for sex and Wilcoxon's rank sum test with continuity correction for age, disease duration, and the UPDRS III scores.

\subsubsection{Comparison of Normalization Procedures, Biomarkers, and Algo- rithms}

We compared BAs between normalization, biomarker, and classification methods in the replication cohort using multiple factor analysis (MFA) as a datadriven exploratory technique and repeated-measures analysis of variance (RM-ANOVA). For the MFA, a first analysis was run by grouping the data by normalization $\times$ biomarkers generating six blocks ( 2 normalizations $\times 3$ biomarkers) of four variables (four algorithms) each 
to determine which "normalization $\times$ biomarker" blocks induced the same structures. A second analysis was run on four blocks (four algorithms) of six variables (2 normalizations $\times 3$ biomarkers) to identify similarities/dissimilarities between algorithms performances. As a first step, individual principal component analysis was performed on each block, which was then normalized by the corresponding first eigenvalue. The obtained matrices were merged to form a global matrix, and a global principal component analysis was performed. The individual observations were then projected onto the global space.

We used RM-ANOVA with a $2 \times 4 \times 3$ factorial design [normalization (1 and 2) $\times$ algorithm (logistic regression, linear SVM, radial SVM, random forest) $\times$ biomarker (volumetry, volumetry + DTI, DTI)] after testing data for normality with ShapiroWilk test $(p>0.05)$. Sphericity assumption was checked using Mauchly's test and, if necessary, corrected using Greenhouse-Geisser correction. Pairwise comparisons with Bonferroni correction were then performed.

\section{Results}

\subsection{Participants' Clinical and Demographic Characteristics}

In total, 322 subjects were analyzed, divided into a training cohort $(n=179)$ and a replication cohort $(n=143)$ (Table 1$)$. In the training cohort, there was a significant difference in sex distribution (Fisher's test, $p=0.031$ ) due to a difference between HCs and patients with $\mathrm{PD}(p=0.040)$. There was no significant difference in age and disease duration between groups. Patients with PD had lower UPDRS III scores than other patient groups $(p<0.002)$. In the replication cohort, there was a difference in age (Kruskal-Wallis, $p<0.002$ ), with patients with PSP being older than other groups. There were no differences in sex distribution, disease duration, and UPDRS III scores between groups.

When comparing both cohorts, there was a significant difference in sex distribution for patients with PSP $(p=0.042)$ and age, because HCs $(p=0.007)$, patients with PD $(p=0.002)$, and patients with PSP $(p=0.019)$ were older in the replication cohort. Disease duration was shorter in the replication cohort for patients with $\mathrm{PD}(p<0.001)$, MSA-P $(p<0.001)$, and MSA-C $(p=0.020)$, but not for patients with PSP $(p=0.055)$. UPDRS III scores were lower for MSA-P $(p=0.003)$ and MSA-C $(p=0.002)$ in the replication cohort.

\subsection{Comparison of Normalization Methods}

The first MFA showed that the first two components with eigenvalues greater than 1 explained $56 \%$ and $20 \%$ of the total variance. Both normalizations with volumetry and normalization 2 with volumetry + DTI strongly correlated with the first dimension, 
Table 1: Demographic and clinical characteristics of the population.

\begin{tabular}{|c|c|c|c|c|c|c|c|c|c|c|}
\hline \multirow[b]{2}{*}{ Groups } & \multicolumn{5}{|c|}{ Training cohort $(n=179)$} & \multicolumn{5}{|c|}{ Replication cohort $(n=143)$} \\
\hline & $\mathrm{HC}$ & $\mathrm{PD}$ & PSP & MSA-P & MSA-C & $\mathrm{HC}$ & $\mathrm{PD}$ & PSP & MSA-P & MSA-C \\
\hline$n$ & 72 & 63 & 21 & 11 & 12 & 22 & 56 & 30 & 24 & 11 \\
\hline Sex ratio $(M / F)$ & $24 / 48^{a}$ & $37 / 26$ & $10 / 11^{b}$ & $4 / 7$ & $7 / 5$ & $12 / 10$ & $36 / 20$ & $23 / 7$ & $18 / 6$ & $7 / 4$ \\
\hline Age at MRI scan (year) & $60.8 \pm 8.2^{c}$ & $60.7 \pm 9.7 \mathrm{c}$ & $65.6 \pm 9.1^{c}$ & $62.8 \pm 7.1$ & $60.3 \pm 7.4$ & $64.7 \pm 7.3$ & $66.6 \pm 11.4$ & $71.7 \pm 5.7^{d}$ & $63.0 \pm 7.0$ & $58.3 \pm 7.7$ \\
\hline Disease duration (year) & NA & $6.0 \pm 4.0^{e}$ & $4.2 \pm 1.9$ & $5.0 \pm 1.9^{e}$ & $5.2 \pm 1.8^{e}$ & NA & $4.2 \pm 3.4$ & $3.4 \pm 2.0$ & $3.8 \pm 1.8$ & $2.4 \pm 1.1$ \\
\hline UPDRS III scores ${ }^{f}$ & $0.4 \pm 0.7$ & $21.0 \pm 13.1^{g}$ & $38.5 \pm 15.7$ & $42.2 \pm 13.9^{h}$ & $47.4 \pm 14.4 \mathrm{~g}$ & $0.1 \pm 0.3$ & $20.6 \pm 9.6$ & $29.6 \pm 17.6$ & $26.3 \pm 10.8$ & $16.7 \pm 7.7$ \\
\hline
\end{tabular}

Group demographics and clinical scores within each cohort were compared. Statistical difference in sex distribution was evaluated using Fisher's exact test, followed by pairwise comparisons with $p$ adjustment (Holm's method). Age, disease duration, and UPDRS III scores were compared using Kruskal-Wallis test, followed by pairwise comparisons with Holm-Bonferroni correction. Inter-cohort comparisons were also performed by assessing group-wise differences using Fisher's exact test for sex and Wilcoxon's rank sum test with continuity correction for age, disease duration, and UPDRS III scores.

Abbreviations: HC, healthy control subjects; PD, Parkinson's disease; PSP, progressive supranuclear palsy; MSA-P, parkinsonian variant of multiple system atrophy; MSA-C, cerebellar variant of multiple system atrophy; M, male; F, female; MRI, magnetic resonance imaging; UPDRS III, Unified Parkinson's Disease Rating Scale Part III.

${ }^{a} p<0.05$ for HCs vs patients with PD in the training cohort.

${ }^{b} p<0.05$ for patients with PSP between both cohorts.

${ }^{c} p<0.05$ for HCs, patients with PD, and patients with PSP between both cohorts.

${ }^{d} p<0.05$ for patients with PSP vs HCs and patients with PD; $p<0.001$ for patients with PSP vs MSA-P and MSA-C in the replication cohort.

$e^{e} p<0.001$ for PD and MSA-P groups between both cohorts; $p<0.05$ for patients with MSA-C.

$f$ UPDRS III scores were missing in $20.2 \%$ of the participants.

${ }^{g} p<0.002$ for PD vs PSP, MSA-P, and MSA-C in the training cohort.

${ }^{h} p<0.05$ for patients with MSA-P and MSA-C between both cohorts. 
and thus showed the same profiles. Normalization 2 with DTI also correlated with the first dimension but less strongly. Normalization 1 with DTI and normalization 1 with volumetry + DTI had a different profile and did not perform as well as the other combinations (Supporting Information Figs. S2 and S3).

RM-ANOVA showed a significant effect of the normalization factor with higher performance of normalization $2(p<0.001)$ and no interactions with the biomarker and algorithm factors. Thus, only results with normalization 2 are provided in the following paragraphs (see Supporting Information Table S4 for results with normalization 1).

\subsection{Comparisons of Biomarkers}

The highest mean BA was observed for volumetry (mean BA $=0.803$ ) followed by volumetry + DTI (mean BA = 0.756) and DTI (mean BA = 0.631). RM-ANOVA with sphericity correction showed a significant effect of biomarkers $(p=0.03)$ and a trend for an algorithm $\times$ biomarkers interaction $(p=0.050)$. Paired t-tests confirmed that volumetry had similar performances to that of volumetry + DTI $(p=0.1)$, and that both performed better than DTI alone $(p<0.001)$. Therefore, DTI underperformed compared with volumetry and did not improve classification when combined with volumetry. Additional MFA did not reveal any significant difference between analyses performed with and without patients with missing DTI. Therefore, imputing the data did not have a significant effect on the overall analysis (Supporting Information Table S6 and Fig. S4). Performances obtained with UPDRS III scores alone were low in both cohorts (BA: 0.397-0.861, AUC: $0.473-0.899$ in the training cohort; BA: $0.316-0.656$, AUC: $0.567-0.659$ in the replication cohort) (Supporting Information Table S7).

\subsection{Comparison of Algorithms}

The second MFA showed that logistic regression, linear SVM, and random forest had similar performances and outperformed radial SVM (Supporting Information Fig. S5). The highest mean BA was observed for logistic regression (mean BA $=0.768$ ), followed by random forest $($ mean $\mathrm{BA}=0.754)$, linear $\mathrm{SVM}$ (mean $\mathrm{BA}=0.745)$, and radial SVM (mean BA $=0.652)$. RM-ANOVA with sphericity correction showed a trend for an algorithm effect $(p=0.055)$ and a trend for a normalization $\times$ algorithm interaction $(p=0.051)$. Post hoc t-tests showed that radial SVM underperformed compared with the other three algorithms that had similar performances. In the following paragraphs, only the results obtained with the logistic regression are reported because it provided the highest mean BA. Results with the three other algorithms are provided in Supporting Information Table S5. 


\subsection{Results per Group Comparison With Normalization 2 and Logistic Regression}

In the training cohort, using volumetry, the best classification performances were obtained in decreasing order for PSP vs MSA-C, PD vs MSA-C, PD vs PSP, PD vs atypical parkinsonian syndromes, and PD vs MSA-P (BA: $0.892-0.963$, AUC: $0.928-0.997$ ). The performances were lower for the classification of PD vs PSP vs MSA (BA: 0.807), PSP vs MSA-P, and MSA-C vs MSA-P (BA: $0.668-0.732$, AUC: $0.757-0.858$ ). Combining volumetry and DTI did not tend to improve BAs $(0.622-0.942)$ or AUCs (0.723 - 0.998), while performances with DTI alone were lower (BA: $0.373-0.880$, AUC: $0.243-0.967$ ) (Table 2).

In the replication cohort, using volumetry, performances remained high for the classifications of PD vs MSA-C, PSP vs MSA-C, PD vs PSP, and PD vs atypical parkinsonism (BA: $0.840-0.983$, AUC: $0.907-0.995)$. Better accuracies than in the training cohort were obtained for the comparisons of PSP vs MSA-P (BA: 0.896, AUC: 0.968) and MSA-C vs MSA-P (BA: 0.784, AUC: 0.871). Performances were lower for the classifications of PD vs MSA-P (BA: 0.765, AUC: 0.839 ) and PD vs PSP vs MSA (BA: 0.773). Classification accuracy using DTI alone was significantly lower (BA: $0.560-0.917$, AUC: $0.731-0.982)$ than volumetry alone $(p<0.001)$, whereas volumetry + DTI (BA: $0.777-0.982$, AUC: $0.833-0.994)$ did not differ from volumetry alone $(p=0.1)$ (Table 2).

\subsection{Weighting Factors}

The best features to differentiate PD and PSP were the volumes of the midbrain (1) and third ventricle $(-0.94)$, FA in the SCP $(0.72)$, and the volumes of the globus pallidus (0.71) and the putamen (0.63). For discriminating subjects with MSA-C, the most relevant features were the midbrain/pons ratio (vs PD and PSP: -1, MSA-P: 1) and the pons atrophy (vs PD: 0.61, MSA-P: -0.47 , PSP: 0.31). The other features were the volumes of the fourth ventricle (vs PD: -0.46 ) and the cerebellum (vs PD: 0.49, PSP: 0.22, MSA-P: -0.36). MSA-P differentiation relied mostly on the putamen volume $(0.46-1)$, with the contribution of DTI metrics in the putamen being low (Table 3 and Figure 1).

\section{Discussion}

Our study demonstrates the feasibility of an automated classification of parkinsonian syndromes in a clinical setting using a large cohort of patients (Archer et al., 2019; Huppertz et al., 2016). The classification algorithms were tested on a large independent replication cohort comprising patients recruited in a movement disorder clinic and scanned using different MRI systems as part of their routine diagnostic workup. Over- 
Table 2: Performances of the logistic regression classification in both cohorts using normalization 2, with volumetry, DTI, and the combination of volumetry and DTI.

\begin{tabular}{|c|c|c|c|c|c|c|c|}
\hline & \multirow[b]{2}{*}{ Metric } & \multicolumn{3}{|c|}{ Training cohort } & \multicolumn{3}{|c|}{ Replication cohort } \\
\hline & & Vol & DTI & Vol + DTI & Vol & DTI & $\mathrm{Vol}+\mathrm{DTI}$ \\
\hline \multirow[t]{4}{*}{$P D$ vs $P S P$} & BA & $0.892(0.090)$ & $0.794(0.103)$ & $0.892(0.094)$ & 0.840 & 0.644 & 0.879 \\
\hline & $\mathrm{AUC}$ & $0.977(0.033)$ & $0.885(0.104)$ & $0.974(0.033)$ & 0.968 & 0.739 & 0.943 \\
\hline & $\mathrm{Se}$ & $0.850(0.189)$ & $0.695(0.197)$ & $0.840(0.187)$ & 0.733 & 0.467 & 0.900 \\
\hline & $\mathrm{Sp}$ & $0.934(0.066)$ & $0.892(0.091)$ & $0.945(0.062)$ & 0.946 & 0.821 & 0.857 \\
\hline \multirow[t]{4}{*}{ PD vs MSA-P } & $\mathrm{BA}$ & $0.845(0.127)$ & $0.800(0.190)$ & $0.880(0.133)$ & 0.765 & 0.679 & 0.744 \\
\hline & AUC & $0.928(0.096)$ & $0.863(0.167)$ & $0.975(0.044)$ & 0.839 & 0.749 & 0.847 \\
\hline & $\mathrm{Se}$ & $0.909(0.069)$ & $0.931(0.081)$ & $0.969(0.044)$ & 0.821 & 0.857 & 0.946 \\
\hline & $\mathrm{Sp}$ & $0.780(0.251)$ & $0.670(0.373)$ & $0.790(0.269)$ & 0.708 & 0.500 & 0.542 \\
\hline \multirow[t]{4}{*}{$\mathrm{PD}$ vs $\mathrm{MSA}-\mathrm{C}$} & $\mathrm{BA}$ & $0.963(0.088)$ & $0.798(0.177)$ & $0.928(0.112)$ & 0.982 & 0.902 & 0.982 \\
\hline & $\mathrm{AUC}$ & $0.991(0.026)$ & $0.948(0.070)$ & $0.992(0.023)$ & 0.995 & 0.966 & 0.994 \\
\hline & $\mathrm{Se}$ & $0.995(0.024)$ & $0.977(0.045)$ & $0.995(0.018)$ & 0.964 & 0.804 & 0.964 \\
\hline & $\mathrm{Sp}$ & $0.930(0.175)$ & $0.620(0.358)$ & $0.860(0.227)$ & 1.000 & 1.000 & 1.000 \\
\hline \multirow[t]{4}{*}{ PSP vs MSA-C } & $\mathrm{BA}$ & $0.938(0.084)$ & $0.880(0.100)$ & $0.942(0.092)$ & 0.983 & 0.917 & 0.967 \\
\hline & $\mathrm{AUC}$ & $0.997(0.016)$ & $0.967(0.058)$ & $0.998(0.012)$ & 0.991 & 0.982 & 0.994 \\
\hline & $\mathrm{Se}$ & $0.970(0.082)$ & $0.900(0.152)$ & $0.990(0.049)$ & 0.967 & 0.833 & 0.933 \\
\hline & $\mathrm{Sp}$ & $0.907(0.166)$ & $0.860(0.179)$ & $0.893(0.184)$ & 1.000 & 1.000 & 1.000 \\
\hline \multirow[t]{4}{*}{ PSP vs MSA-P } & $\mathrm{BA}$ & $0.732(0.181)$ & $0.833(0.155)$ & $0.828(0.156)$ & 0.896 & 0.750 & 0.833 \\
\hline & $\mathrm{AUC}$ & $0.858(0.149)$ & $0.966(0.066)$ & $0.962(0.075)$ & 0.968 & 0.814 & 0.892 \\
\hline & $\mathrm{Se}$ & $0.784(0.180)$ & $0.896(0.135)$ & $0.896(0.147)$ & 0.833 & 0.833 & 0.833 \\
\hline & $\mathrm{Sp}$ & $0.680(0.331)$ & $0.770(0.307)$ & $0.760(0.307)$ & 0.958 & 0.667 & 0.833 \\
\hline \multirow[t]{4}{*}{ MSA-C vs MSA-P } & $\mathrm{BA}$ & $0.668(0.152)$ & $0.373(0.172)$ & $0.622(0.179)$ & 0.784 & 0.725 & 0.788 \\
\hline & $\mathrm{AUC}$ & $0.757(0.203)$ & $0.243(0.185)$ & $0.723(0.217)$ & 0.871 & 0.811 & 0.871 \\
\hline & $\mathrm{Se}$ & $0.630(0.282)$ & $0.320(0.346)$ & $0.590(0.345)$ & 0.750 & 0.542 & 0.667 \\
\hline & $\mathrm{Sp}$ & $0.707(0.239)$ & $0.427(0.337)$ & $0.653(0.260)$ & 0.818 & 0.909 & 0.909 \\
\hline \multirow{4}{*}{$\begin{array}{l}\text { PD vs atypical } \\
\text { parkinsonism }\end{array}$} & BA & $0.879(0.073)$ & $0.742(0.089)$ & $0.880(0.077)$ & 0.853 & 0.658 & 0.839 \\
\hline & $\mathrm{AUC}$ & $0.948(0.051)$ & $0.832(0.097)$ & $0.947(0.053)$ & 0.907 & 0.731 & 0.905 \\
\hline & $\mathrm{Se}$ & $0.903(0.083)$ & $0.840(0.098)$ & $0.934(0.062)$ & 0.875 & 0.732 & 0.893 \\
\hline & $\mathrm{Sp}$ & $0.856(0.113)$ & $0.644(0.174)$ & $0.827(0.139)$ & 0.831 & 0.585 & 0.785 \\
\hline \multirow[t]{4}{*}{$\mathrm{PD}$ vs $\mathrm{PSP}$ vs $\mathrm{MSA}^{a}$} & $\mathrm{BA}$ & $0.807(0.071)$ & $0.711(0.100)$ & $0.812(0.078)$ & 0.773 & 0.560 & 0.777 \\
\hline & $\mathrm{Se}(\mathrm{PD})$ & $0.906(0.080)$ & $0.842(0.100)$ & $0.912(0.081)$ & 0.875 & 0.750 & 0.911 \\
\hline & Se (MSA) & $0.684(0.145)$ & $0.672(0.218)$ & $0.708(0.171)$ & 0.743 & 0.629 & 0.686 \\
\hline & $\mathrm{Se}(\mathrm{PSP})$ & $0.830(0.176)$ & $0.620(0.201)$ & $0.815(0.172)$ & 0.700 & 0.300 & 0.733 \\
\hline
\end{tabular}

For the training cohort, we have reported means and standard deviations (in parentheses) of performance metrics across all repetitions during the cross-validation procedure.

Abbreviations: Vol, volumetry; DTI, diffusion tensor imaging; PD, Parkinson's disease; PSP, progressive supranuclear palsy; BA, balanced accuracy; AUC, area under the curve; Se, sensitivity; Sp, specificity; MSA-P, parkinsonian variant of multiple system atrophy; MSA-C, cerebellar variant of multiple system atrophy; HC, healthy control subjects.

${ }^{a}$ For the multi-class classification task (PD vs PSP vs MSA), in addition to BAs, we have reported sensitivities, that is, the proportion of subjects who have been accurately classified for each class. For instance, $\mathrm{Se}(\mathrm{PD})=$ 0.80 means that $80 \%$ of the patients with PD have been classified as PD by the algorithm. 
Table 3: Weighting factors extracted from the logistic regression training for the different group comparisons using normalization 2.

\begin{tabular}{|c|c|c|c|c|c|c|c|}
\hline & $\begin{array}{c}\text { PD } \\
\text { vs } \\
\text { PSP }\end{array}$ & $\begin{array}{c}\text { PD } \\
\text { vs } \\
\text { MSA-P }\end{array}$ & $\begin{array}{c}\text { PD } \\
\text { vs } \\
\text { MSA-C }\end{array}$ & $\begin{array}{c}\text { PSP } \\
\text { vs } \\
\text { MSA-C }\end{array}$ & $\begin{array}{c}\text { PSP } \\
\text { vs } \\
\text { MSA-P }\end{array}$ & $\begin{array}{l}\text { MSA-C } \\
\text { vs } \\
\text { MSA-P }\end{array}$ & $\begin{array}{c}\mathrm{PD} \\
\mathrm{vs} \\
\text { atypical } \\
\text { parkinsonism }\end{array}$ \\
\hline Midbrain_vol & 1.00 & 0.28 & 0.34 & -0.15 & -0.46 & -0.11 & 1.00 \\
\hline Pons_vol & 0.42 & 0.29 & 0.61 & 0.31 & 0.14 & -0.47 & 0.74 \\
\hline Midbrain/Pons_vol & 0.32 & -0.27 & -1.0 & -1.0 & -1.0 & 1.00 & -0.41 \\
\hline $\mathrm{SCP} \_$vol & 0.37 & 0.16 & 0.25 & 0.04 & -0.04 & -0.13 & 0.48 \\
\hline V3_vol & -0.94 & -0.18 & -0.06 & 0.37 & 0.64 & -0.12 & -0.69 \\
\hline V4_vol & -0.46 & -0.41 & -0.5 & -0.25 & -0.1 & 0.41 & -0.71 \\
\hline Cerebellum_vol & 0.49 & 0.35 & 0.49 & 0.22 & 0.07 & -0.36 & 0.81 \\
\hline Thalamus_vol & 0.59 & 0.22 & 0.07 & -0.21 & -0.24 & 0.18 & 0.36 \\
\hline Caudate_vol & 0.12 & 0.33 & 0.02 & -0.04 & 0.20 & 0.24 & 0.17 \\
\hline Putamen_vol & 0.63 & 1.00 & 0.20 & -0.09 & 0.46 & 0.57 & 0.98 \\
\hline Pallidum_vol & 0.71 & 0.51 & 0.17 & -0.17 & 0.03 & 0.36 & 0.83 \\
\hline Insula_vol & -0.02 & 0.04 & 0.03 & 0.04 & 0.05 & -0.05 & -0.15 \\
\hline Precentral_vol & 0.24 & 0.25 & -0.01 & -0.12 & 0.03 & 0.23 & 0.06 \\
\hline Midbrain_FA & 0.40 & 0.0 & 0.13 & -0.05 & -0.2 & -0.06 & 0.24 \\
\hline Pons_FA & 0.30 & 0.15 & 0.18 & 0.05 & 0.06 & -0.03 & 0.20 \\
\hline $\mathrm{SCP}_{\text {_FA }}$ & 0.72 & 0.17 & 0.11 & -0.22 & -0.44 & 0.06 & 0.56 \\
\hline Putamen_FA & -0.25 & -0.23 & -0.11 & 0.0 & -0.11 & -0.09 & -0.43 \\
\hline Posteriorputamen_FA & -0.13 & -0.14 & -0.11 & -0.04 & -0.04 & 0.06 & -0.25 \\
\hline Pallidum_FA & 0.01 & 0.0 & 0.02 & 0.0 & -0.03 & 0.0 & 0.13 \\
\hline Thalamus_FA & 0.15 & -0.11 & -0.03 & -0.09 & -0.33 & -0.11 & -0.15 \\
\hline Caudate_FA & -0.01 & 0.08 & 0.07 & 0.05 & 0.0 & -0.08 & 0.07 \\
\hline Cerebellum_FA & 0.21 & -0.11 & 0.13 & 0.02 & -0.23 & -0.21 & -0.04 \\
\hline Insula_FA & 0.01 & 0.03 & 0.11 & 0.06 & -0.03 & -0.14 & -0.02 \\
\hline Precentral_FA & 0.25 & 0.07 & 0.10 & -0.03 & -0.21 & -0.11 & 0.26 \\
\hline Midbrain_MD & -0.33 & 0.01 & -0.11 & 0.04 & 0.20 & 0.10 & -0.2 \\
\hline Pons_MD & -0.04 & -0.08 & -0.19 & -0.18 & -0.26 & 0.12 & -0.05 \\
\hline $\mathrm{SCP}_{-} \mathrm{MD}$ & -0.22 & -0.56 & -0.31 & -0.21 & -0.52 & -0.01 & -0.43 \\
\hline Putamen_MD & -0.06 & 0.07 & -0.05 & -0.01 & 0.06 & 0.06 & -0.07 \\
\hline Posteriorputamen_MD & 0.0 & -0.01 & -0.13 & -0.09 & -0.05 & 0.11 & -0.12 \\
\hline Pallidum_MD & 0.04 & 0.21 & 0.08 & 0.05 & 0.17 & 0.02 & 0.11 \\
\hline Thalamus_MD & -0.03 & 0.18 & 0.10 & 0.09 & 0.23 & 0.03 & 0.20 \\
\hline Caudate_MD & -0.2 & -0.08 & -0.01 & 0.05 & 0.13 & 0.02 & -0.21 \\
\hline Cerebellum_MD & -0.08 & -0.23 & -0.27 & -0.24 & -0.34 & 0.18 & -0.23 \\
\hline Insula_MD & -0.27 & 0.03 & -0.02 & 0.08 & 0.25 & 0.08 & -0.13 \\
\hline Precentral_MD & -0.21 & 0.07 & 0.02 & 0.10 & 0.26 & 0.04 & -0.06 \\
\hline Midbrain_AD & -0.21 & 0.11 & -0.07 & 0.04 & 0.28 & 0.16 & -0.08 \\
\hline Pons_AD & 0.07 & 0.0 & -0.18 & -0.22 & -0.26 & 0.18 & 0.07 \\
\hline $\mathrm{SCP} \_\mathrm{AD}$ & 0.14 & -0.41 & -0.22 & -0.29 & -0.64 & 0.03 & -0.08 \\
\hline Putamen_AD & -0.12 & 0.01 & -0.08 & -0.02 & 0.03 & 0.04 & -0.19 \\
\hline Pallidum_AD & 0.07 & 0.27 & 0.12 & 0.07 & 0.20 & 0.02 & 0.22 \\
\hline Thalamus_AD & 0.0 & 0.20 & 0.11 & 0.08 & 0.22 & 0.03 & 0.22 \\
\hline Caudate_AD & -0.18 & -0.06 & 0.01 & 0.06 & 0.12 & 0.01 & -0.16 \\
\hline Cerebellum_AD & 0.01 & -0.21 & -0.2 & -0.21 & -0.37 & 0.10 & -0.15 \\
\hline Insula_AD & -0.25 & 0.05 & 0.01 & 0.10 & 0.25 & 0.05 & -0.09 \\
\hline Precentral_AD & -0.14 & 0.11 & 0.07 & 0.10 & 0.22 & 0.0 & 0.05 \\
\hline Midbrain_RD & -0.34 & 0.01 & -0.1 & 0.09 & 0.24 & -0.02 & -0.26 \\
\hline Pons_RD & -0.06 & 0.05 & -0.16 & -0.11 & -0.07 & 0.09 & 0.0 \\
\hline $\mathrm{SCP}_{2} \mathrm{RD}$ & -0.46 & -0.43 & -0.23 & 0.01 & -0.03 & -0.08 & -0.46 \\
\hline Putamen_RD & -0.03 & 0.10 & 0.07 & 0.10 & 0.07 & -0.17 & 0.09 \\
\hline Pallidum_RD & 0.05 & 0.30 & 0.18 & 0.14 & 0.20 & -0.11 & 0.34 \\
\hline Thalamus_RD & -0.08 & 0.24 & 0.10 & 0.13 & 0.27 & -0.04 & 0.16 \\
\hline Caudate_RD & -0.28 & -0.02 & -0.02 & 0.11 & 0.22 & -0.02 & -0.28 \\
\hline Cerebellum_RD & -0.22 & -0.23 & -0.43 & -0.29 & -0.25 & 0.30 & -0.45 \\
\hline Insula_RD & -0.36 & -0.04 & 0.02 & 0.19 & 0.25 & -0.17 & -0.25 \\
\hline Precentral_RD & -0.39 & 0.0 & 0.07 & 0.25 & 0.42 & -0.16 & -0.21 \\
\hline
\end{tabular}

Weighting factors were scaled to a range of -1 to +1 . Higher absolute values indicate greater contribution of the feature. When the coefficient was positive, the algorithm favored the first disease group if the value of the feature was high or the second disease group if the value was low. Conversely, when the coefficient was negative, the algorithm favored the second group if the value of the feature was high, or the first group if the value was low. Positive values are highlighted in shades of red while negative values are highlighted in shades of blue.

Abbreviations: PD, Parkinson's disease; PSP, progressive supranuclear palsy; vol, volume; MSA-P, parkinsonian variant of multiple system atrophy; MSA-C, cerebellar variant of multiple system atrophy; SCP, superior cerebellar peduncles; V3, third ventricle; V4, fourth ventricle; FA, fractional anisotropy; MD, mean diffusivity; AD, axial diffusivity; $\mathrm{RD}$, radial diffusivity. 


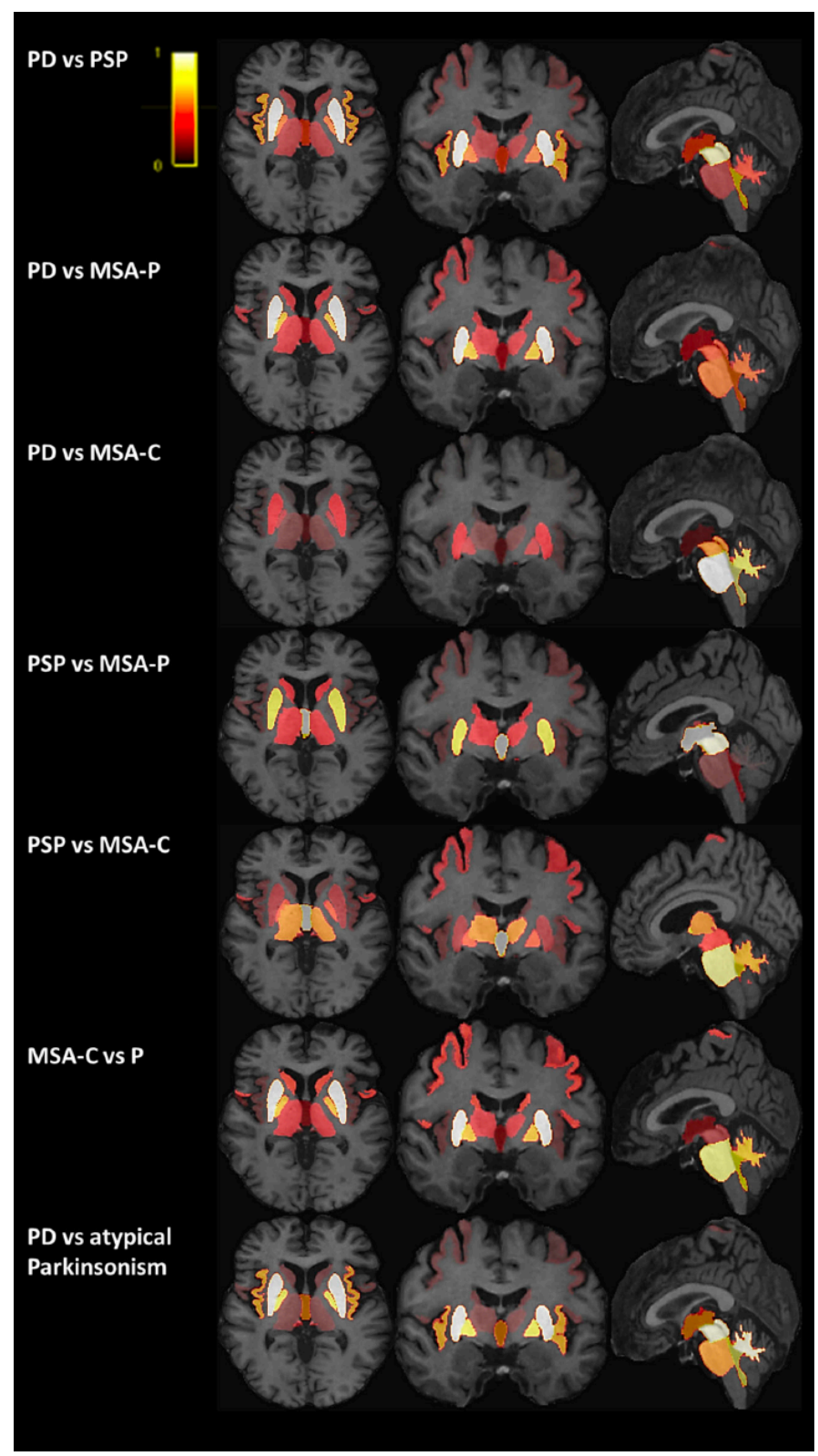

Figure 1: Contribution of each region of interest for the group classification using volumetry. A color shade was attributed to the weighting factor corresponding to the volume for each brain region, depending on its relevance for the group differentiation. A color bar in the top left corner of the figure indicates the shades, with brighter color representing greater weight. Weighting factors were rescaled to the range of 0 to 1 . Weighting factors corresponding to diffusion tensor imaging metrics are not represented. The most relevant regions were Parkinsons disease $(\mathrm{PD})$ versus progressive supranuclear palsy (PSP): midbrain and putamen; $\mathrm{PD}$ versus parkinsonian variant of multiple system atrophy (MSA-P): putamen; PD versus cerebellar variant of multiple system atrophy (MSAC): pons; PSP versus MSA-P: midbrain and third ventricle; PSP versus MSA-C: pons and third ventricle; MSA-P versus MSA-C: putamen and pons; and PD versus atypical parkinsonism: putamen, midbrain, cerebellum, and fourth ventricle. 
all, patients in the replication cohort had a shorter disease duration than those in the training cohort, suggesting that the algorithm could differentiate between patients with early to moderately advanced parkinsonism. A further strength is that patients with both parkinsonian and cerebellar subtypes of MSA were included in addition to PD and PSP, which was only done in two previous studies (Huppertz et al., 2016; Morisi et al., 2018).

Our results are in agreement with previous studies using machine learning to differentiate parkinsonian syndromes and reporting BAs between from $69 \%$ and $89 \%$ (Huppertz et al., 2016), and AUC greater than 93\% (Archer et al., 2019), and 95\% (Scherfler et al., 2016). Nevertheless, algorithm performances are difficult to compare across studies given differences in terms of input data, diseases studied, type of classification and performance indices used. Performances of the logistic regression using volumetry were also equivalent to those obtained with automated methods using clinical measurements such as the Magnetic Resonance Parkinsonism Index (MRPI) for the differentiation of PSP-RS from non-PSP participants (Nigro et al., 2020). However, we found lower accuracy for the classification of MSA-P versus PD and MSA-C and for the multi-class classification. Indeed, MSA-P and MSA-C patients often have overlapping features, a clinical and brain imaging continuum existing between both variants, which makes them more difficult to distinguish (Castellani, 1998; Dickson, 2018). The lower performance in categorizing MSA-P and MSA-C may also be explained by the fact we included mixed participants with both patterns of MSA in the MSA-P group, and by insufficient algorithm training due to the relative small number of MSA patients in the training cohort.

We confirmed that morphometric measurements are robust MRI biomarkers for the discrimination of parkinsonism (Huppertz et al., 2016; Salvatore et al., 2014; Scherfler et al., 2016). In line with previous pathological and imaging studies (Dickson, 2012; Kassubek, 2018; Levin et al., 2016; Messina et al., 2011; Whitwell et al., 2017), the best features for the differentiation between PD and PSP were the midbrain and third ventricle volumes. The midbrain to pons volume ratio was the most relevant feature for the discrimination between PSP and both MSA variants as reported with manual morphometric indices such as the midbrain to pons area ratio (Mangesius et al., 2018; Möller et al., 2017) and the MRPI (Quattrone et al., 2008). The putamen volume was highly discriminant between MSA-P and PD, MSA-P being characterized by a prominent putamen atrophy as compared to PD (Heim et al., 2018; Kassubek, 2018; Messina et al., 2011; Whitwell et al., 2011), but less relevant for the differentiation with PSP (Messina et al., 2011) and MSA-C patients (Castellani, 1998).

Diffusion measurements in the putamen had low contributions to differentiate MSA$\mathrm{P}$ from other disease groups in our study. Several studies have shown increased diffusivity in the posterior putamen in MSA-P versus PD patients (Pellecchia et al., 2009; Seppi et al., 2006; Tsukamoto et al., 2012). Results are less clear between MSA-P 
and PSP patients with overlapping diffusivity (Focke et al., 2011; Nicoletti et al., 2006; Seppi et al., 2003) and iron content (Focke et al., 2011; Sjöström et al., 2017) reported in the entire putamen. Measurements in the posterior putamen may provide better results (Tsukamoto et al., 2012). In our study, co-registration inaccuracies between $\mathrm{T}_{1}$-weighted images and diffusion maps due to echo planar imaging distortions and susceptibility artifacts could have contributed to reducing the accuracy of the diffusion measurements and the performance of the algorithms.

In the replication cohort, normalization using control data reduced the scanner effect and improved categorization performances using DTI. However, combining DTI to volumetry did not improve performances while DTI alone had lower performances, as assessed by the overall low weighting factors associated with DTI measurements. The heterogeneity of DTI data in the replication cohort, which included images acquired using two MRI systems and heterogeneous acquisition parameters (voxel size, number of directions, geometric distortions), probably explained the lower categorization accuracy. Standardization of DTI acquisition parameters may improve classification performances as suggested previously (Archer et al., 2019).

Our study has several limitations. First, there was no neuropathological confirmation of parkinsonian diagnosis, which is the case in most neurodegenerative studies. Second, our study focused on PSP-RS while the differential diagnosis between PD and PSP-Parkinsonism is also challenging. The recruitment of PSP variants will be the scope of future studies. Third, DTI-derived white matter tract microstructure could have improved categorization performance, especially since these large tracts could be less susceptible to changes in DTI scan resolution. Future work could also involve free-water and free-water-corrected measurements, which may improve classification accuracy (Archer et al., 2019). Furthermore, the automated segmentation and machine learning approach that we used are time-consuming and not yet available in clinical routine unlike morphometric methods such as the MRPI. However, there is a huge interest in machine learning approaches, with a high potential for translation in clinical practice.

To conclude, our study showed that automated categorization of parkinsonian syndromes was applicable to patients with early to moderately advanced parkinsonism recruited in a clinical environment, despite variability in scanners and acquisition parameters. Volumetry was the most robust discriminative biomarker. Improvement in acquisition and analysis of diffusion data and inclusion of iron measurements may improve classification. Implementing a machine learning algorithm in the clinical workflow may thus be relevant to help clinicians improve diagnosis of parkinsonism. 


\section{Acknowledgments}

This work was supported by grants from Agence Nationale de la Recherche [grant numbers: ANR-11-INBS-0006 (France Life Imaging), ANRMNP 2009 (Nucleipark), ANR11-INBS-0011 (NeurATRIS), Investissements dAvenir (ANR-19-P3IA-0001, PRAIRIE 3IA Institute, and ANR-10-IAIHU-06, IHU Paris Institute of Neurosciences)], Association France Parkinson, Ecole Neuroscience de Paris, Électricité de France (Fondation dEntreprise EDF), Institut National de la Santé et de la Recherche Médicale, DHOS-

Inserm (2010, Nucleipark), PSP France, the Fondation Thérèse and René Planiol pour létude du Cerveau, European Union (EU) Framework Project 6GENEPARK, the Programme Hospitalier de Recherche Clinique [grant Numbers: PHRC 2007-A00169-44 (LRRK) and PHRC 2004 (BBBIPPS)], the ICM Big Brain Theory Program (project PredictICD), and the Inria Project Lab Program (project Neuromarkers).

\section{Supporting Data}

Additional Supporting Information may be found in the online version of this article at the publisher's web-site. 


\section{References}

Derek B. Archer, Justin T. Bricker, Winston T. Chu, Roxana G. Burciu, Johanna L. McCracken, Song Lai, Stephen A. Coombes, Ruogu Fang, Angelos Barmpoutis, Daniel M. Corcos, Ajay S. Kurani, Trina Mitchell, Mieniecia L. Black, Ellen Herschel, Tanya Simuni, Todd B. Parrish, Cynthia Comella, Tao Xie, Klaus Seppi, Nicolaas I. Bohnen, Martijn LTM Müller, Roger L. Albin, Florian Krismer, Guangwei Du, Mechelle M. Lewis, Xuemei Huang, Hong Li, Ofer Pasternak, Nikolaus R. McFarland, Michael S. Okun, and David E. Vaillancourt. Development and validation of the automated imaging differentiation

in parkinsonism (AID-P): a multicentre machine learning study. The Lancet Digital Health, 1(5):e222-e231, September 2019. doi: 10.1016/S2589-7500(19) 30105-0. URL https://www.scholars.northwestern.edu/en/publications/ development-and-validation-of-the-automated-imaging-differentiati. Publisher: Elsevier Ltd.

A. Berardelli, G. K. Wenning, A. Antonini, D. Berg, B. R. Bloem, V. Bonifati, D. Brooks, D. J. Burn, C. Colosimo, A. Fanciulli, J. Ferreira, T. Gasser, F. Grandas, P. Kanovsky, V. Kostic, J. Kulisevsky, W. Oertel, W. Poewe, J.-P. Reese, M. Relja, E. Ruzicka, A. Schrag, K. Seppi, P. Taba, and M. Vidailhet. EFNS/MDS-ES/ENS [corrected] recommendations for the diagnosis of Parkinson's disease. European Journal of Neurology, 20(1):16-34, January 2013. ISSN 1468-1331. doi: 10.1111/ene. 12022.

Rudy Castellani. Multiple System Atrophy. The American Journal of Pathology, 153 (3):671-676, September 1998. ISSN 0002-9440. URL https://www.ncbi.nlm.nih. gov/pmc/articles/PMC1852997/.

Andrea Cherubini, Maurizio Morelli, Rita Nisticó, Maria Salsone, Gennarina Arabia, Roberta Vasta, Antonio Augimeri, Maria Eugenia Caligiuri, and Aldo Quattrone. Magnetic resonance support vector machine discriminates between Parkinson disease and progressive supranuclear palsy. Movement Disorders: Official Journal of the Movement Disorder Society, 29(2):266-269, February 2014. ISSN 1531-8257. doi: 10.1002/mds.25737.

Dennis W. Dickson. Parkinsons Disease and Parkinsonism: Neuropathology. Cold Spring Harbor Perspectives in Medicine, 2(8), August 2012. ISSN 2157-1422. doi: 10. 1101/cshperspect.a009258. URL https://www.ncbi.nlm.nih.gov/pmc/articles/ PMC3405828/.

Dennis W. Dickson. Neuropathology of Parkinson disease. Parkinsonism \& Related Disorders, 46 Suppl 1:S30-S33, January 2018. ISSN 1873-5126. doi: 10.1016/j. parkreldis.2017.07.033. 
G. Du, M. M. Lewis, S. Kanekar, N. W. Sterling, L. He, L. Kong, R. Li, and X. Huang. Combined Diffusion Tensor Imaging and Apparent Transverse Relaxation Rate Differentiate Parkinson Disease and Atypical Parkinsonism. American Journal of Neuroradiology, 38(5):966-972, May 2017. ISSN 0195-6108, 1936-959X. doi: 10.3174/ajnr.A5136. URL http://www.ajnr.org/content/38/5/966. Publisher: American Journal of Neuroradiology Section: ADULT BRAIN.

Bruce Fischl, David H. Salat, Evelina Busa, Marilyn Albert, Megan Dieterich, Christian Haselgrove, Andre van der Kouwe, Ron Killiany, David Kennedy, Shuna Klaveness, Albert Montillo, Nikos Makris, Bruce Rosen, and Anders M. Dale. Whole brain segmentation: automated labeling of neuroanatomical structures in the human brain. Neuron, 33(3):341-355, January 2002. ISSN 0896-6273. doi: 10.1016/s0896-6273(02) 00569-x.

N. K. Focke, G. Helms, P. M. Pantel, S. Scheewe, M. Knauth, C. G. Bachmann, J. Ebentheuer, P. Dechent, W. Paulus, and C. Trenkwalder. Differentiation of Typical and Atypical Parkinson Syndromes by Quantitative MR Imaging. American Journal of Neuroradiology, 32(11):2087-2092, December 2011. ISSN 0195-6108, 1936-959X. doi: 10.3174/ajnr.A2865. URL http://www.ajnr.org/content/32/11/2087. Publisher: American Journal of Neuroradiology Section: Brain.

S Gilman, G K. Wenning, P A. Low, D J. Brooks, C J. Mathias, J Q. Trojanowski, N W. Wood, C Colosimo, A Dürr, C J. Fowler, H Kaufmann, T Klockgether, A Lees, W Poewe, N Quinn, T Revesz, D Robertson, P Sandroni, K Seppi, and M Vidailhet. Second consensus statement on the diagnosis of multiple system atrophy. Neurology, 71(9):670-676, August 2008. ISSN 0028-3878. doi: 10.1212/01.wnl.0000324625.00404. 15. URL https://www.ncbi.nlm.nih.gov/pmc/articles/PMC2676993/.

J. J. Hauw, S. E. Daniel, D. Dickson, D. S. Horoupian, K. Jellinger, P. L. Lantos, A. McKee, M. Tabaton, and I. Litvan. Preliminary NINDS neuropathologic criteria for Steele-Richardson-Olszewski syndrome (progressive supranuclear palsy). Neurology, 44(11):2015-2019, November 1994. ISSN 0028-3878. doi: 10.1212/wnl.44.11.2015.

Beatrice Heim, Florian Krismer, and Klaus Seppi. Structural Imaging in Atypical Parkinsonism. International Review of Neurobiology, 142:67-148, 2018. ISSN 21625514. doi: 10.1016/bs.irn.2018.08.010.

Gunter U. Hoglinger, Gesine Respondek, Maria Stamelou, Carolin Kurz, Keith A. Josephs, Anthony E. Lang, Brit Mollenhauer, Ulrich Muller, Christer Nilsson, Jennifer L. Whitwell, Thomas Arzberger, Elisabet Englund, Ellen Gelpi, Armin Giese, David J. Irwin, Wassilios G. Meissner, Alexander Pantelyat, Alex Rajput, John C. van Swieten, Claire Troakes, Angelo Antonini, Kailash P. Bhatia, Yvette Bordelon, Yaroslau Compta, Jean-Christophe Corvol, Carlo Colosimo, Dennis W. Dickson, 
Richard Dodel, Leslie Ferguson, Murray Grossman, Jan Kassubek, Florian Krismer, Johannes Levin, Stefan Lorenzl, Huw R. Morris, Peter Nestor, Wolfgang H. Oertel, Werner Poewe, Gil Rabinovici, James B. Rowe, Gerard D. Schellenberg, Klaus Seppi, Thilo van Eimeren, Gregor K. Wenning, Adam L. Boxer, Lawrence I. Golbe, and Irene Litvan. Clinical Diagnosis of Progressive Supranuclear Palsy: The Movement Disorder Society Criteria. Movement disorders : official journal of the Movement Disorder Society, 32(6):853-864, June 2017. ISSN 0885-3185. doi: 10.1002/mds.26987. URL https://www.ncbi.nlm.nih.gov/pmc/articles/PMC5516529/.

Andrew J. Hughes, Susan E. Daniel, Yoav Ben-Shlomo, and Andrew J. Lees. The accuracy of diagnosis of parkinsonian syndromes in a specialist movement disorder service. Brain: A Journal of Neurology, 125(Pt 4):861-870, April 2002. ISSN 00068950. doi: 10.1093/brain/awf080.

Hans-Jürgen Huppertz, Leona Möller, Martin Südmeyer, Rüdiger Hilker, Elke Hattingen, Karl Egger, Florian Amtage, Gesine Respondek, Maria Stamelou, Alfons Schnitzler, Elmar H. Pinkhardt, Wolfgang H. Oertel, Susanne Knake, Jan Kassubek, and Günter U. Höglinger. Differentiation of neurodegenerative parkinsonian syndromes by volumetric magnetic resonance imaging analysis and support vector machine classification. Movement Disorders: Official Journal of the Movement Disorder Society, 31(10):1506-1517, 2016. ISSN 1531-8257. doi: 10.1002/mds.26715.

Lorraine V. Kalia and Anthony E. Lang. Parkinson's disease. The Lancet, 386(9996):896-912, August 2015. ISSN 0140-6736, 1474-547X. doi: 10.1016/ S0140-6736(14)61393-3. URL https://www.thelancet.com/journals/lancet/ article/PIIS0140-6736(14)61393-3/abstract. Publisher: Elsevier.

Jan Kassubek. MRI-based neuroimaging: atypical parkinsonisms and other movement disorders. Current Opinion in Neurology, 31(4):425-430, 2018. ISSN 1473-6551. doi: 10.1097/WCO.0000000000000578.

Stéphane Lehericy, David E. Vaillancourt, Klaus Seppi, Oury Monchi, Irena Rektorova, Angelo Antonini, Martin J. McKeown, Mario Masellis, Daniela Berg, James B. Rowe, Simon J. G. Lewis, Caroline H. Williams-Gray, Alessandro Tessitore, Hartwig R. Siebner, and International Parkinson and Movement Disorder Society (IPMDS)Neuroimaging Study Group. The role of high-field magnetic resonance imaging in parkinsonian disorders: Pushing the boundaries forward. Movement Disorders: Official Journal of the Movement Disorder Society, 32(4):510-525, 2017. ISSN 1531-8257. doi: $10.1002 /$ mds.26968.

Johannes Levin, Alexander Kurz, Thomas Arzberger, Armin Giese, and Günter U. Höglinger. The Differential Diagnosis and Treatment of Atypical Parkinsonism. 
Deutsches Arzteblatt International, 113(5):61-69, February 2016. ISSN 1866-0452. doi: 10.3238/arztebl.2016.0061.

I. Litvan, Y. Agid, D. Calne, G. Campbell, B. Dubois, R. C. Duvoisin, C. G. Goetz, L. I. Golbe, J. Grafman, J. H. Growdon, M. Hallett, J. Jankovic, N. P. Quinn, E. Tolosa, and D. S. Zee. Clinical research criteria for the diagnosis of progressive supranuclear palsy (Steele-Richardson-Olszewski syndrome): report of the NINDSSPSP international workshop. Neurology, 47(1):1-9, July 1996. ISSN 0028-3878. doi: 10.1212/wnl.47.1.1.

Stephanie Mangesius, Anna Hussl, Florian Krismer, Philipp Mahlknecht, Eva Reiter, Susanne Tagwercher, Atbin Djamshidian, Michael Schocke, Regina Esterhammer, Gregor Wenning, Christoph Müller, Christoph Scherfler, Elke R. Gizewski, Werner Poewe, and Klaus Seppi. MR planimetry in neurodegenerative parkinsonism yields high diagnostic accuracy for PSP. Parkinsonism 6 Related Disorders, 46: 47-55, January 2018. ISSN 1353-8020. doi: 10.1016/j.parkreldis.2017.10.020. URL http://www.sciencedirect.com/science/article/pii/S1353802017304212.

Demetrio Messina, Antonio Cerasa, Francesca Condino, Gennarina Arabia, Fabiana Novellino, Giuseppe Nicoletti, Maria Salsone, Maurizio Morelli, Pier Luigi Lanza, and Aldo Quattrone. Patterns of brain atrophy in Parkinson's disease, progressive supranuclear palsy and multiple system atrophy. Parkinsonism \& Related Disorders, 17(3):172-176, March 2011. ISSN 1873-5126. doi: 10.1016/j.parkreldis.2010.12.010.

Rita Morisi, David Neil Manners, Giorgio Gnecco, Nico Lanconelli, Claudia Testa, Stefania Evangelisti, Lia Talozzi, Laura Ludovica Gramegna, Claudio Bianchini, Giovanna Calandra-Buonaura, Luisa Sambati, Giulia Giannini, Pietro Cortelli, Caterina Tonon, and Raffaele Lodi. Multi-class parkinsonian disorders classification with quantitative MR markers and graph-based features using support vector machines. Parkinsonism \& Related Disorders, 47:64-70, 2018. ISSN 1873-5126. doi: 10.1016/j.parkreldis.2017.11.343.

Leona Möller, Jan Kassubek, Martin Südmeyer, Rüdiger Hilker, Elke Hattingen, Karl Egger, Florian Amtage, Elmar H. Pinkhardt, Gesine Respondek, Maria Stamelou, Franz Möller, Alfons Schnitzler, Wolfgang H. Oertel, Susanne Knake, Hans-Jürgen Huppertz, and Günter U. Höglinger. Manual MRI morphometry in Parkinsonian syndromes. Movement Disorders: Official Journal of the Movement Disorder Society, 32(5):778-782, 2017. ISSN 1531-8257. doi: 10.1002/mds.26921.

Giuseppe Nicoletti, Raffaele Lodi, Francesca Condino, Caterina Tonon, Francesco Fera, Emil Malucelli, David Manners, Mario Zappia, Letterio Morgante, Paolo Barone, Bruno Barbiroli, and Aldo Quattrone. Apparent diffusion coefficient measurements of the middle cerebellar peduncle differentiate the Parkinson variant of 
MSA from Parkinson's disease and progressive supranuclear palsy. Brain: A Journal of Neurology, 129(Pt 10):2679-2687, October 2006. ISSN 1460-2156. doi: $10.1093 /$ brain/awl166.

Salvatore Nigro, Angelo Antonini, David E. Vaillancourt, Klaus Seppi, Roberto Ceravolo, Antonio P. Strafella, Antonio Augimeri, Andrea Quattrone, Maurizio Morelli, Luca Weis, Eleonora Fiorenzato, Roberta Biundo, Roxana G. Burciu, Florian Krismer, Nikolaus R. McFarland, Christoph Mueller, Elke R. Gizewski, Mirco Cosottini, Eleonora Del Prete, Sonia Mazzucchi, and Aldo Quattrone. Automated MRI Classification in Progressive Supranuclear Palsy: A Large International Cohort Study. Movement Disorders, 35(6):976-983, 2020. ISSN 1531-8257. doi: https://doi.org/ 10.1002/mds.28007. URL https://onlinelibrary •wiley.com/doi/abs/10 .1002/ mds.28007. _eprint: https://onlinelibrary.wiley.com/doi/pdf/10.1002/mds.28007.

Fabian Pedregosa, Gaël Varoquaux, Alexandre Gramfort, Vincent Michel, Bertrand Thirion, Olivier Grisel, Mathieu Blondel, Peter Prettenhofer, Ron Weiss, Vincent Dubourg, Jake Vanderplas, Alexandre Passos, David Cournapeau, Matthieu Brucher, Matthieu Perrot, and Édouard Duchesnay. Scikit-learn: Machine Learning in Python. Journal of Machine Learning Research, 12(85):2825-2830, 2011. ISSN 1533-7928. URL http://jmlr.org/papers/v12/pedregosa11a.html.

Maria Teresa Pellecchia, Paolo Barone, Carmine Mollica, Elena Salvatore, Marta Ianniciello, Katia Longo, Andrea Varrone, Caterina Vicidomini, Marina Picillo, Giuseppe De Michele, Alessandro Filla, Marco Salvatore, and Sabina Pappatà. Diffusionweighted imaging in multiple system atrophy: a comparison between clinical subtypes. Movement Disorders: Official Journal of the Movement Disorder Society, 24 (5):689-696, April 2009. ISSN 1531-8257. doi: 10.1002/mds.22440.

Nadya Pyatigorskaya, Lydia YahiaCherif, Rahul Gaurav, Claire Ewenczyk, Cecile Gallea, Romain Valabregue, Fatma Gargouri, Benoit Magnin, Bertrand Degos, Emmanuel Roze, Eric Bardinet, Cyril Poupon, Isabelle Arnulf, Marie Vidailhet, and Stéphane Lehericy. Multimodal Magnetic Resonance Imaging Quantification of Brain Changes in Progressive Supranuclear Palsy. Movement Disorders, 35(1):161-170, 2020. ISSN 1531-8257. doi: https://doi.org/10.1002/mds.27877. URL https://onlinelibrary.wiley.com/doi/abs/10.1002/mds.27877. _eprint: https://onlinelibrary.wiley.com/doi/pdf/10.1002/mds.27877.

Patrice Péran, Gaetano Barbagallo, Federico Nemmi, Maria Sierra, Monique Galitzky, Anne Pavy-Le Traon, Pierre Payoux, Wassilios G. Meissner, and Olivier Rascol. MRI supervised and unsupervised classification of Parkinson's disease and multiple system atrophy. Movement Disorders: Official Journal of the Movement Disorder Society, 33(4):600-608, 2018. ISSN 1531-8257. doi: 10.1002/mds.27307. 
Aldo Quattrone, Giuseppe Nicoletti, Demetrio Messina, Francesco Fera, Francesca Condino, Pierfrancesco Pugliese, Pierluigi Lanza, Paolo Barone, Letterio Morgante, Mario Zappia, Umberto Aguglia, and Olivier Gallo. MR imaging index for differentiation of progressive supranuclear palsy from Parkinson disease and the Parkinson variant of multiple system atrophy. Radiology, 246(1):214-221, January 2008. ISSN 1527-1315. doi: 10.1148/radiol.2453061703.

Giovanni Rizzo, Stefano Zanigni, Roberto De Blasi, Daniela Grasso, Davide Martino, Rodolfo Savica, and Giancarlo Logroscino. Brain MR Contribution to the Differential Diagnosis of Parkinsonian Syndromes: An Update. Parkinson's Disease, 2016: 2983638, 2016. ISSN 2090-8083. doi: 10.1155/2016/2983638.

C. Salvatore, A. Cerasa, I. Castiglioni, F. Gallivanone, A. Augimeri, M. Lopez, G. Arabia, M. Morelli, M. C. Gilardi, and A. Quattrone. Machine learning on brain MRI data for differential diagnosis of Parkinson's disease and Progressive Supranuclear Palsy. Journal of Neuroscience Methods, 222:230-237, January 2014. ISSN 1872-678X. doi: 10.1016/j.jneumeth.2013.11.016.

Christoph Scherfler, Georg Göbel, Christoph Müller, Michael Nocker, Gregor K. Wenning, Michael Schocke, Werner Poewe, and Klaus Seppi. Diagnostic potential of automated subcortical volume segmentation in atypical parkinsonism. Neurology, 86(13): 1242-1249, March 2016. ISSN 1526-632X. doi: 10.1212/WNL.0000000000002518.

K. Seppi, M. F. H. Schocke, R. Esterhammer, C. Kremser, C. Brenneis, J. Mueller, S. Boesch, W. Jaschke, W. Poewe, and G. K. Wenning. Diffusion-weighted imaging discriminates progressive supranuclear palsy from $\mathrm{PD}$, but not from the parkinson variant of multiple system atrophy. Neurology, 60(6):922-927, March 2003. ISSN 1526-632X. doi: 10.1212/01.wnl.0000049911.91657.9d.

Klaus Seppi, Michael F. H. Schocke, Kathrin Prennschuetz-Schuetzenau, Katherina J. Mair, Regina Esterhammer, Christian Kremser, Armin Muigg, Christoph Scherfler, Werner Jaschke, Gregor K. Wenning, and Werner Poewe. Topography of putaminal degeneration in multiple system atrophy: a diffusion magnetic resonance study. Movement Disorders: Official Journal of the Movement Disorder Society, 21(6):847-852, June 2006. ISSN 0885-3185. doi: 10.1002/mds.20843.

Henrik Sjöström, Tobias Granberg, Eric Westman, and Per Svenningsson. Quantitative susceptibility mapping differentiates between parkinsonian disorders. Parkinsonism \&6 Related Disorders, 44:51-57, November 2017. ISSN 1873-5126. doi: 10.1016/j. parkreldis.2017.08.029.

John C. Steele, J. Clifford Richardson, and Jerzy Olszewski. Progressive supranuclear palsy: a heterogeneous degeneration involving the brain stem, Basal Ganglia and 
cerebellum with vertical gaze and pseudobulbar palsy, nuchal dystonia and dementia. Seminars in Neurology, 34(2):129-150, April 2014. ISSN 1098-9021. doi: 10.1055/ s-0034-1377058.

Kazumichi Tsukamoto, Eiji Matsusue, Yoshiko Kanasaki, Suguru Kakite, Shinya Fujii, Toshio Kaminou, and Toshihide Ogawa. Significance of apparent diffusion coefficient measurement for the differential diagnosis of multiple system atrophy, progressive supranuclear palsy, and Parkinson's disease: evaluation by 3.0-T MR imaging. Neuroradiology, 54(9):947-955, September 2012. ISSN 1432-1920. doi: 10.1007/s00234-012-1009-9.

Jennifer L. Whitwell, Ankit V. Master, Ramesh Avula, Kejal Kantarci, Scott D. Eggers, Heidi A. Edmonson, Clifford R. Jack, and Keith A. Josephs. Clinical correlates of white matter tract degeneration in PSP. Archives of Neurology, 68(6):753-760, June 2011. ISSN 0003-9942. doi: 10.1001/archneurol.2011.107. URL https://www.ncbi. nlm.nih.gov/pmc/articles/PMC3401587/.

Jennifer L. Whitwell, Günter U. Höglinger, Angelo Antonini, Yvette Bordelon, Adam L. Boxer, Carlo Colosimo, Thilo van Eimeren, Lawrence I. Golbe, Jan Kassubek, Carolin Kurz, Irene Litvan, Alexander Pantelyat, Gil Rabinovici, Gesine Respondek, Axel Rominger, James B. Rowe, Maria Stamelou, Keith A. Josephs, and Movement Disorder Society-endorsed PSP Study Group. Radiological biomarkers for diagnosis in PSP: Where are we and where do we need to be? Movement Disorders: Official Journal of the Movement Disorder Society, 32(7):955-971, July 2017. ISSN 1531-8257. doi: $10.1002 /$ mds.27038. 